\title{
Tratamento endovascular de pseudoaneurisma de aorta torácica: relato de caso
}

\author{
Endovascular treatment of thoracic aortic pseudoaneurysm: case report \\ Luis Carlos Mendes de Brito ${ }^{1}$, João de Toledo Martins ${ }^{1}$, Ovanil Furlani Júnior ${ }^{2}$, \\ Sérgio Penteado de Camargo Oliveira Júnior ${ }^{3}$, Azis Arruda Chagury ${ }^{4}$, \\ Karina Ribeiro Cavalcante Tavares ${ }^{4}$
}

\begin{abstract}
Resumo
Paciente de 31 anos vítima de acidente automobilístico apresentou falta de ar inspiratória progressiva acompanhada de dor dissecante no tórax, de forte intensidade, sem irradiação; Glasgow 15 e ISS 26 (16+9+ 1); exames laboratoriais e radiográficos compatíveis com a normalidade. Foi operado de fratura de tíbia 1 dia depois, tendo recebido alta 3 dias depois da entrada no serviço. No entanto, foi piorando da falta de ar e da dor torácica, retornando para nova consulta 2 dias após a alta hospitalar. Foi realizada tomografia computadorizada helicoidal que revelou pseudoaneurisma de aorta torácica. Optou-se por tratamento endovascular com implante de endoprótese.
\end{abstract} torácicos.

Palavras-chave: Pseudoaneurisma, aorta torácica, traumatismos

\section{Introdução}

O trauma tem sido apontado como a principal causa de morte na população adulta jovem no mundo, e o traumatismo torácico, em especial, tem se destacado em virtude de fatores vinculados aos avanços tecnológicos, aos meios de transporte cada vez mais rápidos e ao aumento da violência urbana $^{1-3}$. O trauma de tórax pode ser classificado em fechado ou penetrante em função da abertura ou não da cavidade pleural. Qualquer um dos órgãos contidos na cavidade torácica pode ser atingido durante um trauma, dependendo do mecanismo, trajetória e magnitude des$\mathrm{te}^{4}$. Uma grave consequência do trauma de tórax é o pseudoaneurisma ou falso aneurisma de aorta, que é uma entidade clínica causada por ruptura de uma das camadas da parede arterial, podendo ser retida pelos tecidos vizinhos.

\begin{abstract}
A 31-year-old car accident victim was admitted with progressive inspiratory dyspnea, followed by intense dissecting thoracic pain, without any irradiation. The patient's Glasgow score was 15 , while his ISS was $26(16+9+1)$. Laboratory and radiographic results were normal. One day after hospital admission, the patient underwent surgery due to a fractured tibia, being discharged from hospital 3 days later. However, worsening shortness of breath and thoracic pain were observed and he returned to the hospital 2 days later. A helical computed tomography was done revealing a thoracic aortic pseudoaneurysm. Endovascular surgery with endoprosthesis implantation was the treatment of choice.
\end{abstract}

Keywords: Pseudoaneurysm, thoracic aorta, thoracic injuries.

Os autores apresentam um relato de caso de traumatismo torácico fechado e desaceleração brusca causados por colisão de automóvel que culminou com pseudoaneurisma de aorta torácica, optando-se por tratamento endovascular com implante de endoprótese no Serviço de Hemodinâmica de Sorocaba. O interesse deste caso reside na raridade da incidência e da sobrevida dos pacientes com este tipo de lesão, visto que a maioria dos casos vai a óbito antes do atendimento hospitalar, e no bom resultado do tratamento devido ao emprego de nova técnica minimamente invasiva em comparação às técnicas de tratamento convencionais.

\section{Apresentação do caso \\ Paciente do sexo masculino, 31 anos, vítima de aci- dente automobilístico, apresentou falta de ar inspiratória gradativa e sem fatores de melhora acompanhada de dor}

\footnotetext{
1. Cirurgião vascular, Radiologia Vascular Intervencionista, Sorocaba, SP.

2. Cirurgião vascular, Sorocaba, SP.

3. Cirurgião torácico, Sorocaba, SP.

4. Acadêmicos de Medicina, Centro de Ciências Médicas e Biológicas, Pontifícia Universidade Católica de São Paulo, Sorocaba, SP.

Não foram declarados conflitos de interesse associados à publicação deste artigo.

Artigo submetido em 28.08.08, aceito em 26.11.08.

J Vasc Bras. 2010;9(1):57-60.

Copyright (C 2010 by Sociedade Brasileira de Angiologia e de Cirurgia Vascular
} 
dissecante no tórax, de forte intensidade, sem irradiação. No exame físico apresentava-se em regular estado geral, levemente descorado e com murmúrio vesicular, sem ruídos adventícios. Fratura em tíbia esquerda. Exames laboratoriais e radiográficos compatíveis com a normalidade. Operado da fratura de tíbia 1 dia depois, recebendo alta 3 dias depois da entrada no serviço. No entanto, foi piorando da falta de ar e da dor torácica, retornando para nova consulta 2 dias após a alta hospitalar. Realizou-se tomografia computadorizada (TC) helicoidal (Figura 1) que revelou arco aórtico com dilatação focal e sinais de dissecção localizada com formação de imagem sacular na aorta descendente com 3,0 cm de diâmetro e 2,0 cm de extensão, havendo dupla luz.

No mesmo dia, optou-se pelo tratamento endovascular. Inicialmente, foi puncionada a artéria femoral comum esquerda e colocado introdutor valvulado 5F. Passou-se cateter guia Pig Tail centimetrado e foi realizada a angiografia que confirmou o diagnóstico de pseudoaneurisma da aorta torácica (Figura 2A). Realizou-se inguinotomia direita com isolamento da artéria femoral comum e, logo depois, punção da artéria femoral comum direita e passagem de fio guia Lunderquist ${ }^{\circledR}$ de $0,038 ", 260 \mathrm{~cm}$, posicionando-se este no arco aórtico. Através desse fio foi posicionada e liberada a endoprótese Talent ${ }^{\circledR} 34 / 34 / 115 \mathrm{~mm}$ (Figura 2B) e foi realizada a acomodação da endoprótese com balão de látex. Em seguida, uma angiografia após o implante da endoprótese evidenciou a exclusão do pseudoaneurisma e revelou a aorta torácica pérvia em toda a sua extensão.

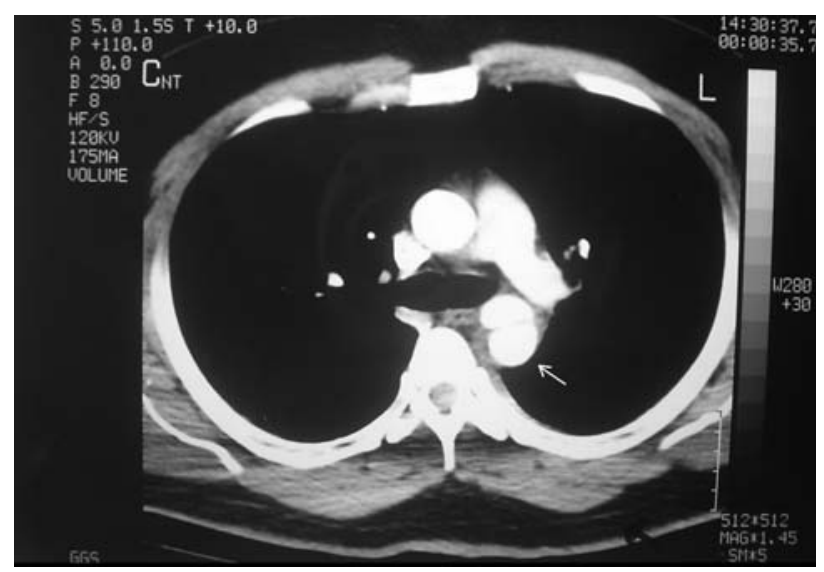

Figura 1 - Tomografia computadorizada 5 dias após acidente. Evidencia-se aorta torácica com dupla luz. Corte transversal
O paciente recebeu alta 3 dias após o procedimento, sendo realizada nova tomografia que demonstrou exclusão da falsa luz e estabilização do quadro clínico. O paciente foi acompanhado e retornou em 30 dias (Figura 3A) e em 90 dias (Figura 3B) para TC de controle que revelou desaparecimento completo do pseudoaneurisma e também dos sintomas referidos anteriormente.

\section{Discussão}

O tratamento cirúrgico convencional de aneurisma de aorta exige toracotomia e substituição da aorta torácica por uma prótese. Com o tratamento endovascular, a toracoto-
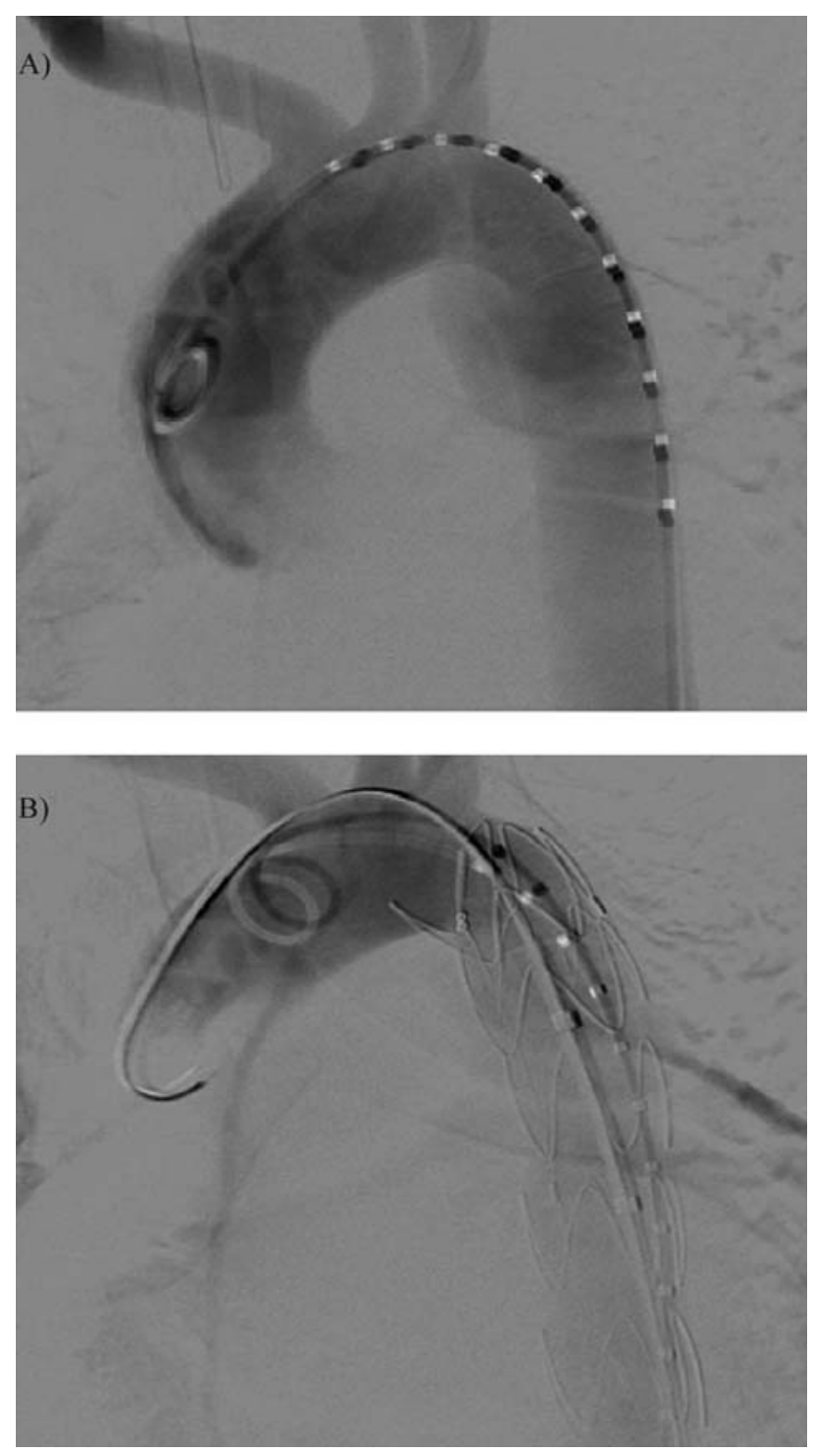

Figura 2 - A) Angiografia revelando pseudoaneurisma em aorta descendente; B) angiografia evidenciando o posicionamento da endoprótese em aorta descendente 

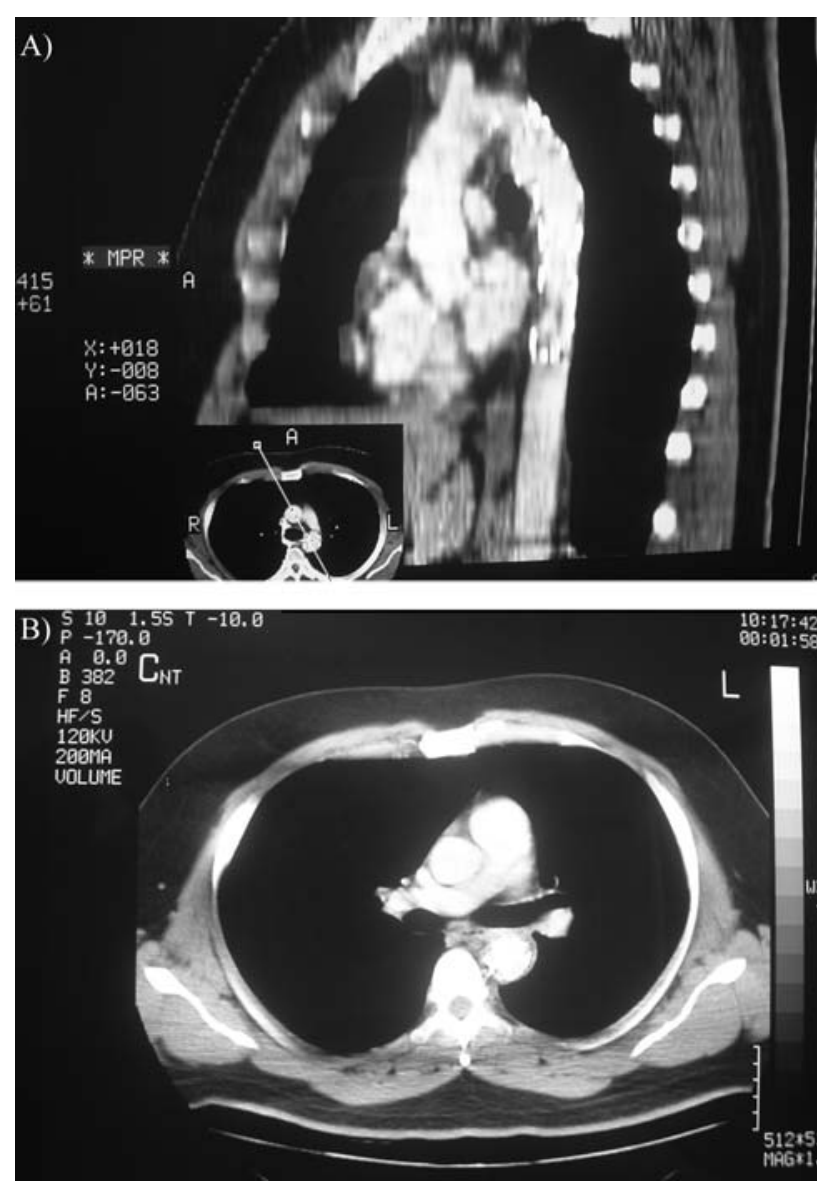

Figura 3 - A) Tomografia computadorizada de controle após 30 dias do procedimento, mostrando desaparecimento da dupla luz; B) tomografia computadorizada de controle após 90 dias do procedimento: endoprótese bem posicionada e sem evidência de pseudoaneurisma

mia é evitada e, por incisões na região inguinal, a endoprótese pode ser implantada mediante um procedimento menos invasivo. A endoprótese é avançada através de guias, retrogradamente, pela artéria femoral comum, via artérias ilíacas, até a aorta abdominal e, consequentemente, aorta torácica. Uma vez posicionada adequadamente, é liberada imediatamente. Desse modo, a perda sanguínea é bem menor do que a produzida com a cirurgia convencional, a aorta não precisa ser pinçada e a recuperação do paciente é mais rápida. Vários estudos prospectivos e randomizados estão sendo conduzidos no sentido de comparar a técnica convencional com a endovascular ${ }^{5-10}$. Dois deles, o EVAR $1^{11}$ e o DREAM ${ }^{12}$, demonstram tendência a menor mortalidade cirúrgica, mesmo em pacientes de baixo risco, oferecendo uma alternativa viável e com menor morbidade em relação à cirurgia convencional. Além disso, Ott et al. relataram um menor risco de complicações pós-operatórias, como infarto do miocárdio, sepse, tempo de ventilação mecânica e síndrome de desconforto respiratório agudo, em pacientes com lesões traumáticas de aorta tratados pelo método endovascular. Nesse mesmo estudo, com o método aberto obteve-se $17 \%$ de mortalidade ( $\mathrm{n}=$ 2), $16 \%$ de paraplegia $(n=2)$ e $8,3 \%(n=1)$ de lesão do nervo laringo-recorrente; já com a cirurgia endovascular, não houve nenhuma dessas complicações ${ }^{13}$.

Em outro estudo, feito com 1904 pacientes, foram observados melhores índices do tratamento endovascular em relação à cirurgia aberta nos dados referentes à mortalidade em 30 dias $(3,1 \%$ versus $5,6 \%, \mathrm{p}=0.01)$, mortalidade após 1 ano $(8,7$ versus $12.1 \%)$ e riscos perioperatórios ( 15,5 versus $27,7 \%)^{14}$. Já no Brasil, apesar de não haverem muitos estudos multicêntricos comparativos, houve melhora significativa da morbidade e de complicações pós-operatórias no trauma torácico desde a adoção do tratamento endovascur $\operatorname{lar}^{15,16}$. Nos últimos anos, a utilização de stents tem proporcionado uma nova perspectiva no tratamento das dissecções e dos aneurismas de aorta. A utilização desta técnica permitiu menor tempo de internação hospitalar, diminuição da mortalidade cirúrgica e melhor recuperação pós-operatória, além da diminuição dos gastos hospitalares ${ }^{17,18}$.

Em relação aos efeitos pós-cirúrgicos, um recente estudo mostrou que após 2 anos e 6 meses da cirurgia emergencial, em pacientes com trauma de aorta descendente, não houve interferência quanto à qualidade de vida e aos níveis de depressão e ansiedade ${ }^{19}$.

No que se refere à escolha da prótese para o procedimento, estudos mostraram não haver diferença significativa entre os modelos utilizados $\left(\right.$ Talent ${ }^{\mathbb{B}}$, Excluder $^{\circledR} \mathrm{e}$ Zenith $\left.^{\circledR}\right)^{10,20}$.

Por fim, é necessário refinamento de técnica para permitir a colocação do stent através de introdutores e cateteres menores, sendo importante também, nesse sentido, a boa habilidade do cirurgião. Em contrapartida, com este método podemos evitar complicações inerentes à manipulação cirúrgica convencional como, por exemplo, insuficiência respiratória e renal, coagulopatias, dor pós-operatória, paraplegia, hematoma próximo à prótese e pseudoaneurismas nas zonas de sutura e infecções localizadas e sistêmicas. 


\section{Conclusão}

As vítimas de trauma torácico fechado com acometimento de aorta evidenciam altos índices de morbidade e mortalidade em todo o mundo. Seu diagnóstico é de difícil realização e necessita-se uma equipe bem treinada e preparada no atendimento dessas vítimas.

Com o aprimoramento da cirurgia endovascular e da habilidade do cirurgião, cada vez mais essa técnica vêm apresentando melhores resultados a curto e médio prazos, podendo ser realizada de forma rápida, segura e com baixa morbidade; além disso, ela permite o tratamento de outras lesões associadas, diminui as complicações pós-operatórias e reduz o tempo de internação.

\section{Referências}

1. Giannini JA, Soldá SC, Saad Júnior R. Trauma de tórax. In: Coimbra RSM, Soldá SC, Casaroli AA, et al. Emergências traumáticas e não traumáticas. São Paulo: Atheneu; 2001. p. 57-69.

2. LoCicero J 3rd, Mattox KL. Epidemiology of chest trauma. Surg Clin North Am. 1989;69:15-9.

3. Calhoon JH, Trinkle JK. Pathophysiology of chest trauma. Chest Surg Clin N Am. 1997;7:199-211.

4. Cuba RM, Bezerra JA. Traumatismo torácico: estudo retrospectivo de 168 casos. Rev Col Bras Cir. 2005;32:57-9.

5. Bansal V, Lee J, Coimbra R. Current diagnosis and management of blunt traumatic rupture of the thoracic aorta. J Vasc Bras. 2007;6:64-73.

6. Buz S, Zipfel B, Mulahasanovic S, Pasic M, Weng Y, Hetzer R. Conventional surgical repair and endovascular treatment of acute traumatic aortic rupture. Eur J Cardiothorac Surg. 2008;33:143-9.

7. Tang GL, Tehrani HY, Usman A, et al. Reduced mortality, paraplegia, and stroke with stent graft repair of blunt aortic transections: a modern meta-analysis. J Vasc Surg. 2008;47:671-5.

8. Walsh SR, Tang TY, Sadat U, et al. Endovascular stenting versus open surgery for thoracic aortic disease: systematic review and meta-analysis of perioperative results. J Vasc Surg. 2008;47:1094-8.

9. Moainie SL, Neschis DG, Gammie JS, et al. Endovascular stenting for traumatic aortic injury: an emerging new standard of care. Ann Thorac Surg. 2008;85:1625-9.

10. Orend KH, Zarbis N, Schelzig H, Halter G, Lang G, Sunder-Plassmann L. Endovascular treatment (EVT) of acute traumatic lesions of the descending thoracic aorta: 7 years' experience. Eur J Vasc Endovasc Surg. 2007;34:666-72.

11. EVAR Trial Participants. Endovascular aneurysm repair versus open repair in patients with abdominal aortic aneurysms
(EVAR 1 Trial): Randomized controlled trial. Lancet. 2005;365:2179-86.

12. Blankensteijn JD, de Jong SE, Prinssen M, et al. Two-year outcomes after conventional or endovascular repair of abdominal aortic aneurysms. $\mathrm{N}$ Engl J Med. 2005;352:2398-405

13. Ott MC, Stewart TC, Lawlor DK, Gray DK, Forbes TL. Management of blunt thoracic aortic injuries: endovascular stents versus open repair. J Trauma. 2004;56:565-70.

14. Bown MJ, Sutton AJ, Bell PR, Sayers RD. A meta-analysis of 50 years of ruptured abdominal aortic aneurysm repair. Br J Surg. 2002;89:714-30.

15. Aun R. Ruptura traumática da aorta por traumatismo torácico fechado. J Vasc Bras. 2007;6:5-6.

16. Mioto Neto B, Aun R, Estenssoro AE, Puech-Leão P. Tratamento das lesões de aorta nos traumatismos torácicos fechados. J Vasc Bras. 2005;4:217-26

17. Bush RL, Johnson ML, Collins TC, et al. Open versus endovascular abdominal aortic aneurysm repair in VA hospitals. J Am Coll Surg. Apr 2006;202:577-87.

18. Saratzis N, Melas N, Saratzis A, Lazaridis J, Kiskinis D. Minimally invasive endovascular intervention in emergent and urgent thoracic aortic pathologies: single center experience. Hellenic J Cardiol. 2008;49:312-9.

19. Dick F, Hinder D, Immer FF, et al. Outcome and quality of life after surgical and endovascular treatment of descending aortic lesions. Ann Thorac Surg. 2008;85:1605-12.

20. Mohan IV, Hitos K, White GH, et al. Improved outcomes with endovascular stent grafts for thoracic aorta transections. Eur J Vasc Endovasc Surg. 2008;36:152-7.

Correspondência:

Azis Arruda Chagury

Rua Martinica, 874, Jardim América

CEP 18046-805 - Sorocaba, SP

Tel.: (15) 8126.1096, (15) 3221.5060

E-mail: azischagury@gmail.com

\section{Contribuições dos autores}

Concepção e desenho do estudo: LCMB, AAC

Análise e interpretação dos dados: LCMB, AAC

Coleta de dados: LCMB, AAC, KRCT

Redação do artigo: LCMB, AAC

Revisão crítica do texto: LCMB, JTM, OF Junior Aprovação final do artigo*: LCMB, AAC, JTM, OF Junior, KRCT, SPCO Junior

Análise estatística: N/A

Responsabilidade geral pelo estudo: LCMB

Informações sobre financiamento: N/A

* Todos os autores leram e aprovaram a versão final submetida ao J Vasc Bras. 